\title{
Coherent and Incoherent Drifting Pulse Dynamics in a Complex Ginzburg-Landau Equation
}

\author{
Martin van Hecke, Ewald de Wit, and Wim van Saarloos \\ Instituut-Lorentz, Leiden University, P.O. Box 9506, 2300 RA Leiden, The Netherlands
}

(Received 21 April 1995)

\begin{abstract}
We show that drifting pulse solutions of a 1D complex Ginzburg-Landau equation can persist for positive growth rate $\varepsilon$ in a finite system. When $\varepsilon$ is increased, two different destabilization scenarios are observed. In sufficiently large systems, fluctuations grow out to form multiple pulses. In small systems, an increase in $\varepsilon$ eventually leads to a competition between fronts and pulses that results in a sharp transition to a state where the drifting pulse leaps forward in an incoherent fashion. Similar behavior is observed in a more realistic model.
\end{abstract}

PACS numbers: $47.20 . \mathrm{Hw}, 03.40 . \mathrm{Gc}, 05.40 .+\mathrm{j}, 47.54 .+\mathrm{r}$

A few years ago, localized or confined traveling wave states were discovered in convection experiments in binary liquids [1-7]. These are states of which the region where the convection occurs does not fill the total experimental cell, but instead attains a well-defined width. The discovery of these states has inspired a considerable amount of theoretical work on pulse solutions of amplitude equations [8-12]. It is by now well established that in experiments in annular geometries a localized traveling wave state drifts slowly when the inhomogeneities of the convection cell are sufficiently small [3]. This drift velocity $v_{p}$ is quite different from the group velocity $v_{\mathrm{gr}}$, and this can be contributed to a slow concentration field $[9,10]$ that "traps" the pulse in its own concentration gradient. The existence of localized states and much of their behavior can be understood in terms of pulse-shaped solutions of a complex Ginzburg-Landau amplitude equation $[8,11]$. The fact that the pulse velocity $v_{p}$ differs so much from $v_{\mathrm{gr}}$ can, however, only be obtained from a more detailed analysis of the coupling of the convection to the slow concentration field $[9,10]$.

In the experiments in an annular geometry $[2,5,6]$, the localized traveling wave states surprisingly persist in a regime where the conducting state $(A=0)$ is completely unstable $(\varepsilon>0)$. This persistence of pulses in an unstable background is usually explained as follows. Since an annular cell is periodic and since the pulse drifts with a velocity $v_{p}$ different from the velocity $v_{\mathrm{gr}}$ with which the fluctuations propagate, the maximum time interval during which a fluctuation can grow before interacting with the pulse is finite and of order $L /\left|v_{\mathrm{gr}}-v_{p}\right|$, where $L$ is the circumference of the cell. Glazier and Kolodner [4] observed that small wave packets that collide with a pulse are annihilated, so it is conceivable [5] that when the growth rate $\varepsilon$ is sufficiently small, fluctuations in a finite cell do not grow strong enough to destroy the pulse state: it is as if the moving pulse sweeps the system clean.

This scenario has never been verified theoretically, as most theoretical work is based on perturbation expansions around integrable limits of Ginzburg-Landau amplitude equations and assumes an infinite domain $[8,11]$. Such an analysis is insensitive to the instability of the background state that occurs when $\varepsilon>0$; this instability is usually implemented ad hoc by simply assuming that a pulse cannot persist for positive $\varepsilon$.

In this paper, we investigate the dynamics of pulses when $\varepsilon>0$ for a model amplitude equation that captures the two main experimental ingredients, i.e., periodic boundary conditions and the difference between $v_{p}$ and $v_{\mathrm{gr}}$. Our findings can be summarized in the phase diagram of Fig. 1, which labels the various types of asymptotic states that arise as a function of the system size $L$ and $\varepsilon$, when the initial state is a single pulse. The main subject of this paper is the sharp transition from single coherent pulse motion in regime I to incoherent pulse motion

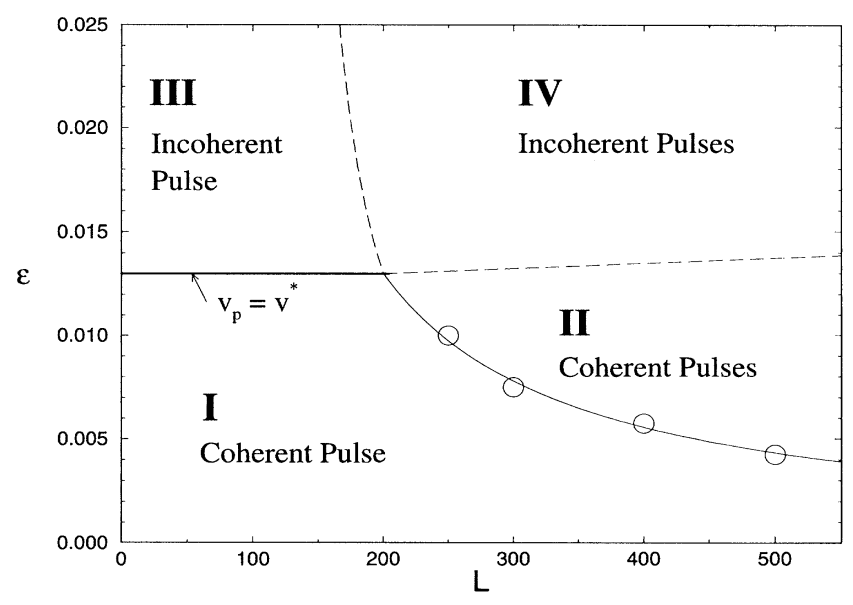

FIG. 1. Tentative phase diagram showing the various states that arise as a function of $\varepsilon$ and $L$. In regimes I and III there are single pulses that either propagate coherently (I) or incoherently (III). Multiple-pulse states are observed in regimes II (coherent) and IV (incoherent). The thin full line marks the transition between these two regimes, and is given by $\varepsilon(L-50)=$ const; the open dots indicate some of the numerical measurements of this transition. The CI transition between the single coherent and incoherent pulses is denoted by a fat line; it occurs at a value $\varepsilon_{\mathrm{CI}}$ given by Eq. (2). The exact location of the transitions between regimes III and IV and between regimes II and IV, indicated by dashed lines, has not been determined.

(C) 1995 The American Physical Society 
in regime III, which we show to be the result of a competition between pulse and front propagation.

The model equation that we study is the so-called quintic derivative Ginzburg-Landau equation $[11,12]$ :

$$
\begin{aligned}
\partial_{t} A= & \varepsilon A+\left(1+i c_{1}\right) \partial_{x}^{2} A+\left(1+i c_{3}\right) A|A|^{2} \\
& -\left(1-i c_{5}\right) A|A|^{4}+s\left(\partial_{x} A\right)|A|^{2} .
\end{aligned}
$$

Although recent work $[6,10]$ demonstrates that a single amplitude equation cannot account for all phenomena observed in the experiments, the essential difference in $v_{\mathrm{gr}}$ and $v_{p}$ and the periodic boundary conditions are captured in this model. Equation (1) is written in the frame of the group velocity of linear waves; the nonlinear gradient term $s\left(\partial_{x} A\right)|A|^{2}$ breaks left-right symmetry and causes pulses to drift. Nonlinear gradient terms of this type arise when a systematic expansion up to fifth order is made. We fix the parameters $c_{1}=1.4, c_{3}=-1$, and $c_{5}=-1$ since pulses are stable then (see Sec. 4.2 of [11]), and fix $s=-1$. For these parameters, a pulse drifts to the right with velocity $v_{p} \approx 1.5 \varepsilon+0.373$ for small $\varepsilon$.

We use a pseudospectral method to numerically solve the amplitude equation (1) with periodic boundary conditions, with a time step of 0.05 and 256 Fourier modes. The main role of fluctuations is to excite the modes that grow from the linearly unstable state $A=0$. We will not systematically study the effect of an additive noise term, which would amount to getting an extra parameter in the phase diagram, since the discretization noise alone is sufficient to excite the unstable modes [13], but we have checked that the inclusion of a stochastic noise term in the amplitude equation (1) or a change in the number of modes qualitatively alters our conclusions. We will proceed by describing the various states that are listed in the phase diagram.

Regime I corresponds to the scenario sketched earlier: the drifting pulse annihilates the fluctuations, and the system behaves the same as for $\varepsilon<0$; this state is the analog of the pulses that persist in the experiments for $\varepsilon>0$. The norm $\mathcal{N} \equiv \int d x|A|$ converges to a value close to the norm of a single pulse in the $\varepsilon<0$ regime.

In regime II the fluctuations grow out to form new pulses before they can be absorbed by the initial pulse. If $L$ and $\varepsilon$ are not too deep into regime II, the system ends up in a state with two pulses. The maximum time interval during which fluctuations can grow without meeting a pulse is then reduced, and therefore the double-pulse state can be stable. The norm converges then to a constant that is approximately twice as big as it is for a singlepulse state. When $L$ and $\varepsilon$ are further increased, states consisting of more pulses are formed that were observed to persist in some cases. Similar behavior was observed by Kolodner [5]. Since the fluctuations grow as $e^{\varepsilon t}$, their maximum strength is roughly determined by the growth rate and the maximum time for which they can grow; in a single-pulse state this time is roughly $(L-W) / v_{p}$, where $W$ is a measure for the width of the pulse that is of order 50 for our choice of parameters. The transition to multiple pulses seems to occur when the fluctuations grow above a certain critical strength, and since $v_{p}$ depends only weakly on $\varepsilon$, the transition curve is expected to be given approximately by $L-W \sim 1 / \varepsilon$ [5], which is the dashed line in Fig. 1. This is in reasonable agreement with our numerics. The inclusion of an additive noise source shifts the transition curve to lower values of $L$ and $\varepsilon$, as one would expect, but there remains a region where the single coherent pulse persists.

The surprise occurs when we cross the border between coherent and incoherent behavior (CI) and enter region III; the motion of the pulse then becomes an irregular mixture of coherent drift and forward leaps. Peaks of the norm as shown in Fig. 3(a) (which we will refer to as "spikes") correspond to a forward leap of the pulse like the one shown in Fig. 2(a). The average time interval $\langle\Delta t\rangle$ between subsequent spikes strongly depends on the distance from the CI transition. This transition can be understood when a connection with the theory of front propagation is made, of which the main ingredients are summarized below.

A perturbation from the unstable state $A=0$ not only grows but also spreads out, due to the diffusive term of the amplitude equation. A single, sufficiently localized perturbation can evolve to a so-called linear marginal stability (LMS) front that connects the unstable equilibrium state with a nonlinear state [14,15]. This front propagates [16] with the LMS velocity $v^{*}$, and its motion is determined

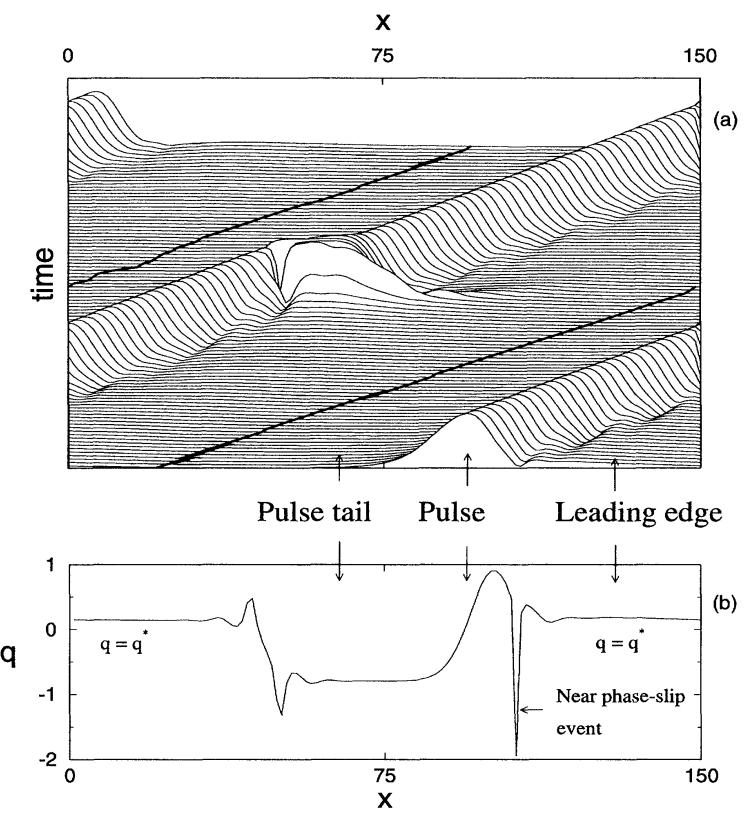

FIG. 2. (a) Space-time plot of $|A|$ of a forward leap event that occurred for $\varepsilon=0.018$ in a system of size 150. Time increases upwards, and every curve in this hidden line plot is separated by a time interval of 5 ; the whole picture occurs over a time interval of 500. The fat curve marks the position in the leading edge of the LMS front where $|A|=0.01$, and propagates with $v^{*}$. (b) A plot of the local wave vector $q$ of $A$ for the initial state of the hidden line plot. 

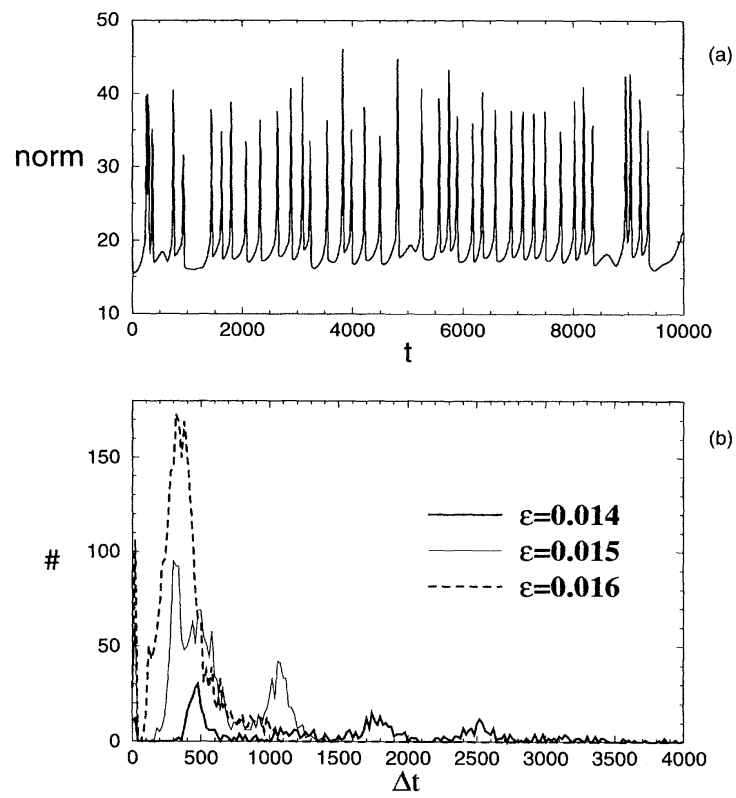

FIG. 3. (a) Typical plot in regime III of the norm $\mathcal{N}$ as a function of time for $\varepsilon=0.018$ and $L=150$. (b) Histogram showing the distribution of the time intervals between two spikes, $\Delta t$, for $\varepsilon=0.014,0.015$, and 0.016 . The system size is 160 , and the total duration of each run $10^{6}$. We have defined the spike to occur when the norm passes through a value of 20 from below, and used a bin of 20 on the time axis.

by its leading edge, which has a well-defined local wave number $q^{*}$, where the local wave number $q$ is defined as $\partial_{x} \arg (A)$. For the amplitude equation (1) the LMS velocity is given by $v^{*}=2 \sqrt{\varepsilon\left(1+c_{1}^{2}\right)}$, and $q^{*}$ is given by $c_{1} \sqrt{\varepsilon /\left(1+c_{1}^{2}\right)}[11,14,15]$.

For our choice of the $c$ 's and large values of $\varepsilon$, localized initial conditions lead to fronts propagating with velocity $v^{*}$; the nonlinear state behind such a front is disordered for our parameters (similar to Fig. 20 of [11]). However, when $v^{*}$ is comparable to the pulse velocity, pulse and front propagation strongly compete, and indeed within our numerical error we find that the CI transition occurs exactly at an $L$-independent value $\varepsilon_{\mathrm{CI}}$ where the front and pulse velocity coincide,

$$
v_{p}\left(\varepsilon_{\mathrm{CI}}\right)=v^{*}\left(\varepsilon_{\mathrm{CI}}\right) \text {. }
$$

For our parameters, this gives $\varepsilon_{\mathrm{CI}} \simeq 0.0130$.

We will now describe our understanding of this result and our evidence supporting our view that this marks the exact $\mathrm{CI}$ transition. Consider again Fig. 2, where we show a space-time plot of $|A|$ to illustrate the dynamics of the single pulse in the incoherent regime III, together with a plot of the local wave number $q$ in the initial state of the space-time plot. After a transient time the fluctuations organize themselves into the structure labeled "leading edge" that propagates ahead of the pulse [17]. The local wave number of this structure is seen from Fig. 2(b) to be close to the theoretical prediction for $q^{*}$, which for $\varepsilon=$
0.018 yields $q^{*} \simeq 0.11$. This fact, together with the fact that this structure only builds up when $v^{*}>v_{p}$, shows that this structure is the leading edge of an LMS front. The evolution of this front is illustrated in Fig. 2(a) by the fat line, which marks the point where $|A|=0.01$; this point propagates with velocity $v^{*}$, and as close inspection of the plot shows, it outruns the pulse: $v^{*}>v_{p}$. After a certain time interval the rear of the LMS front grows out to a nonlinear structure that merges with the pulse. Effectively, the pulse temporarily broadens (leading to a spike in the norm $\mathcal{N}$ ) and then leaps forward to absorb the rear of the LMS structure, while leaving most of the leading edge intact (as evidenced by the absence of appreciable perturbations of the fat line). Then this whole process repeats itself, so that viewed on a long time scale the motion of the pulse can be characterized as a mixture of coherent drift and incoherent forward leaps.

The oscillations that are visible where the right side of the pulse matches onto the LMS front are caused by phase slips that occur because there is a mismatch between the frequency and wave number of the pulse profile and the LMS front. At the initial time shown in the lower panel, such a phase slip event had just occurred. By monitoring singularities in the local wave number, such phase slips can also be observed in the region where the back side of the pulse connects to the leading edge of the LMS front.

A forward leap of the pulse does not seem to affect the leading edge of the LMS front, and so when we are not too deep into the incoherent regime III, the leaps serve to keep the average pulse velocity $\langle v\rangle$ in pace with the front: $\langle v\rangle=v^{*}$ for $\varepsilon>\varepsilon_{\mathrm{CI}}$-see Fig. 4(a). When we denote
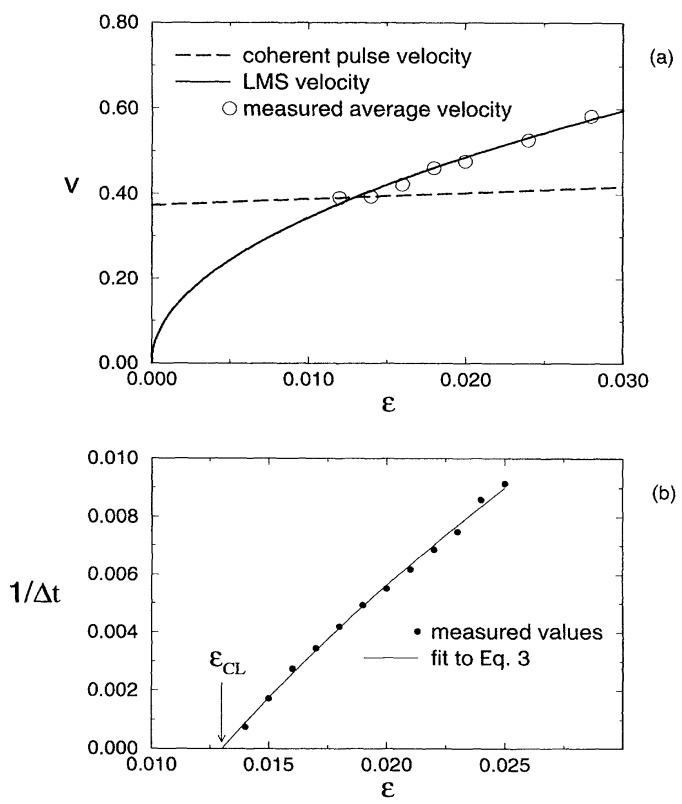

FIG. 4. (a) The LMS velocity $v^{*}$ (full line), the coherent pulse velocity $v_{p}$ (dashed line), and the measured average pulse velocity $\langle v\rangle$ (circles). (b) The average time between two subsequent spikes is seen to diverge as $\varepsilon$ approaches $\varepsilon_{\mathrm{CI}}$. 
the distance of a forward leap by $\Delta x$, then $v^{*}=\langle v\rangle=$ $v_{p}+\langle\Delta x / \Delta t\rangle$. If we assume that $\Delta x$ is a constant, we then obtain

$$
1 /\langle\Delta t\rangle=\left(v^{*}-v_{p}\right) / \Delta x
$$

When we use the aforementioned expressions for $v_{p}$ and $v^{*}$ and fit our data points for $1 /\langle\Delta t\rangle$ by Eq. (3) with $\Delta x$ as a fit parameter, we find a good fit for $\Delta x \simeq 14.8$ as shown in Fig. 4(b); the consistency of the divergence $\Delta t$ as $\varepsilon$ approaches $\varepsilon_{c}$ provides further evidence of the correctness of our scenario for the CI transition.

The distribution of $\Delta t$ close to the CI transition consists of multiple peaks, whereas far from the CI transition only one peak can be observed-see Fig. 3(b); moreover, the location of the first peak of this distribution is not very sensitive to $\varepsilon$. We have at present no explanation for these observations.

It should be noted that the leaps also occur as transient behavior when $v^{*}<v_{p}$. For instance, the evolution from a single to a multipulse state that can be observed when an initial single-pulse state is followed in parameter range II often starts out by the single pulse leaping forward. In this process new pulses are generated that also may perform some leaps, but finally the behavior relaxes to coherent drift in this regime.

Since the two ingredients of our scenario, i.e., the existence of pulses and the linear marginal stability mechanism, are robust, we expect the $\mathrm{CI}$ transition to be rather general. In order to verify this, we have briefly studied the model proposed by Riecke [10] to describe pulses in binary fluid mixtures

$$
\begin{aligned}
& \partial_{t} A= \varepsilon A+\left(1+i c_{1}\right) \partial_{x}^{2} A+\left(1+i c_{3}\right)|A|^{2} A \\
&-\left(1-i c_{5}\right)|A|^{4} A+f C A \\
& \partial_{t} C-v \partial_{x} C=a_{c} C+d_{c} \partial_{x}^{2} C+h \partial_{x}|A|^{2} .
\end{aligned}
$$

$C$ is the real valued concentration field. We have taken the $c$ 's as before and take $f=-0.3, a_{c}=-0.02, d_{c}=$ 0.1 , and $h_{2}=-0.2$. Regimes I, II, and IV can easily be verified to exist. We have found that for $v=0$ and $v=0.1$ the CI transition occurs at values of $\varepsilon$ given by Eq. (2), and that the average speed adjusts to $v^{*}$. However, for increasing $v, \Delta x$ decreases so that regime III shrinks; when $v \geqslant 0.3, \varepsilon_{\mathrm{CI}}$ becomes so large that the pulse destabilizes via a different mechanism. This illustrates that the CI transition is not restricted to Eq. (1), although the detailed dynamics in the incoherent regime may depend on the model. The fact that the amplitude and wave number structure observed numerically (see Fig. 2) are very reminiscent of that seen experimentally is another indication of the robustness of our scenario.
We would like to thank P. C. Hohenberg and P. Kolodner for stimulating comments.

Note added. - After submission of this paper we became aware of the recent work of Chang, Demekhin, and Kopelevich [18], where similar ideas for an extended Kuramoto-Sivashinsky equation play a role.

[1] R. Heinrichs, G. Ahlers, and D. S. Cannell, Phys. Rev. A 35, 2761 (1987); E. Moses, J. Fineberg, and V. Steinberg, Phys. Rev. A 35, 2757 (1987); P. Kolodner, D. Bensimon, and C. M. Surko, Phys. Rev. Lett. 60, 1723 (1988).

[2] J. J. Niemela, G. Ahlers, and D. S. Cannel, Phys. Rev. Lett. 64, 1365 (1990).

[3] P. Kolodner, Phys. Rev. Lett. 66, 1165 (1991).

[4] J. A. Glazier and P. Kolodner, Phys. Rev. A 43, 4269 (1991).

[5] P. Kolodner, Phys. Rev. A 44, 6448 (1991).

[6] P. Kolodner, Phys. Rev. E 50, 2731 (1994).

[7] E. Kaplan, E. Kuznetsov, and V. Steinberg, Europhys. Lett. 28, 237 (1994).

[8] O. Thual and S. Fauve, J. Phys. (Paris) 49, 1829 (1988); B. A. Malomed, Physica (Amsterdam) 29D, 155 (1987); C. Elphick and E. Meron, Phys. Rev. A 40, 3226 (1989); Phys. Rev. Lett. 65, 2476 (1990); V. Hakim, P. Jakobsen, and Y. Pomeau, Europhys. Lett. 11, 19 (1990); B. A. Malomed and A. A. Nepomnyashchy, Phys. Rev. A 42, 6009 (1990); S. Fauve and O. Thual, Phys. Rev. Lett. 64, 282 (1990).

[9] W. Barten, M. Lücke, W. Hort, and M. Kamps, Phys. Rev. Lett. 63, 376 (1989).

[10] H. Riecke, Phys. Rev. Lett. 68, 301 (1992); Physica (Amsterdam) 61D, 253 (1992); H. Herrero and H. Riecke, Physica (Amsterdam) D (to be published).

[11] W. van Saarloos and P. C. Hohenberg, Physica (Amsterdam) 56D, 303 (1992); 69D, 209(E) (1993).

[12] M. C. Cross and P.C. Hohenberg, Rev. Mod. Phys. 65, 851 (1993)

[13] The phase slips that occur even for $\varepsilon<\varepsilon_{\mathrm{CI}}$ in a finite system in the region where the front and back sides of the pulse merge appear to be another source of fluctuations.

[14] E. Ben-Jacob, H. R. Brand, G. Dee, L. Kramer, and J. S. Langer, Physica (Amsterdam) 14D, 348 (1985).

[15] W. van Saarloos, Phys. Rev. A 37, 211 (1988); 39, 6367 (1989).

[16] For the parameters we have chosen, there are no nonlinear front solutions that can overtake the LMS fronts. See [11], in particular, Fig. 8.

[17] Note that "trailing" and "leading" edge refers to the behavior in the frame moving with the group velocity, not to the experimental laboratory frame.

[18] H-C. Chang, E. A. Demekhin, and D. I. Kopelevich, Phys. Rev. Lett. 75, 1747 (1995). 